Forum 2018 $\cdot 33: 66$

https://doi.org/10.1007/s12312-017-0381-0

Online publiziert: 15. Januar 2018

(c) Springer Medizin Verlag $\mathrm{GmbH}$, ein Teil von Springer Nature 2018

\section{Neue Konzepte für eine Nutzenbewertung von morgen}

\section{Sieben Jahre AMNOG-Prozess}

\begin{abstract}
Auch sieben Jahre nach Inkrafttreten des AMNOG halten die Diskussionen um die Ausgestaltung der Nutzenbewertung in der Onkologie an. Beim 3. Symposium zu patientenrelevanten Endpunkten von Springer Medizin, Pfizer und MSD in Berlin tauschten sich onkologische Experten und Vertreter des Gemeinsamen Bundesausschuss (G-BA) intensiv darüber aus, wie ein faires Health Technology Assessment in der Onkologie aussehen sollte.
\end{abstract}

Der deutsche HTA-Prozess berücksichtigt bei seinen Bewertungen bekanntlich Gesamtüberleben (OS), Morbidität, Lebensqualität und Toxizität. Der häufig als primärer Studienendpunkt gewählte Parameter des progressionsfreien Überlebens (PFS) wird hingegen nicht direkt als patientenrelevanter Endpunkt einbezogen. Deutschland stehe mit seiner kategorischen Ablehnung des PFS international sehr isoliert da, sagte Prof. Dr. Diana Lüftner, Berlin. Als aktuelles Beispiel nannte sie den CDK 4/6-Hemmer Palbociclib, dem vom G-BA beim HR-positiven, HER2-negativen, fortgeschrittenen Brustkrebs kein Zusatznutzen beschieden wurde, während das britische NICE Mitte November mit Verweis auf das PFS mitteilte, dass dieses Medikament künftig erstattungsfähig sei.

\section{Aus Patientensicht ist Progression relevant}

Sollte das PFS also auch in der deutschen Nutzenbewertung berücksichtigt werden? Aus Patientensicht unterstrich Doris Schmitt von der Stiftung PATH, dass das PFS für die Lebensqualität selbstverständlich relevant sei. „Es kann nicht sein, dass Frauen von einem Arzneimittel hören, das zugelassen ist und das das PFS verlängert und dann Angst haben müssen, es nicht zu erhalten. "Für den G-BA betonte Dr. Uwe Vosgerau, Berlin, dass der objektive Nachweis besserer Lebensqualität oder eines höheren OS entscheidend bleiben müsse: „Dass ein Progress eine große mentale Belastung sein und die Lebensqualität reduzieren kann, ist nachvollziehbar. Aber dann brauchen wir auch Daten, die das zeigen."

Das PFS ist ein komplexer Parameter, erläuterte Professor Dr. Frank Griesinger, Oldenburg. So gebe es bei der EGFR-Inhibitor-Therapie beim NSCLC dann einen engen Zusammenhang zwischen PFS und OS, wenn der Cross-over-Effekt in Studien berücksichtigt werde. Anders sehe es teilweise bei der Immuntherapie aus. So gab es bei Pembrolizumab bei Patienten mit NSCLC und PD-L1-Expression $\geq 1 \%$ in der Second-Line-Therapie keinen Unterschied gegenüber Docetaxel beim PFS, wohl aber beim OS, was zu einem beträchtlichen Zusatznutzen im AMNOG-Prozess führte. Um der Komplexität von Anti-Tumor- und insbesondere Immuntherapien gerecht $\mathrm{zu}$ werden, müsse daher ein breites Spektrum an Endpunkten betrachtet werden, so Griesinger.

Prof. Lüftner plädierte vor diesem Hintergrund dafür, über alternative Endpunkte nachzudenken. Beispielsweise Zeit bis zur ersten Folgetherapie (TFST) bzw. die Zeit bis zur zweiten Folgetherapie (TSST) könnten das Spektrum der Nutzenbewertungsendpunkte sinnvoll ergänzen.

\section{Registerdaten können ergänzende Informationen liefern}

Viele dieser neuen Endpunkte erfordern systematische Analysen des Verlaufs nach einer Progression. Registerdaten sollten daher künftig stärker für die Nutzenbewertung berücksichtigt werden, sagte PD. Dr. Thomas Zander, Köln. Er sieht hier das Gesundheitswesen als Ganzes in der Pflicht: „Das ist nicht allein Aufgabe der Industrie. “ Um die Dokumentation zu erleichtern, plädierte Zander für eine konsequente Digitalisierung und weitgehende Automatisierung der Erfassung relevanter Parameter: „Auch Patienten sollten stärker in die Dokumentation eingebunden werden", so Zander.

Dr. Antje Behring und Dr. Uwe Vosgerau wiesen für den G-BA darauf hin, dass Registerdaten eine Ergänzung zu RCTs darstellen können - insbesondere klinische Indikationsregister, die gegenüber Produktregistern Vorteile hätten. Als Beispiel wurde das CRISP-Register in der Indikation NSCLC genannt. Inwieweit Registerdaten vor dem Hintergrund schneller Zulassungen und kleiner Patientenpopulationen in der Onkologie eine wertvolle Ergänzung sein könnten, werde seitens des G-BA jeweils individuell bewertet.

Philipp Grätzel von Grätz, Medizinjournalist

\section{Korrespondenzadresse}

Dr. H.-U. Jelitto

Verantwortlicher Pressevertreter der Sektion C in der DKG

Roche Pharma AG

h-u.jelitto@roche.com 